Adjusting for socioeconomic status revealed reduced ORs for mental health problems in the Pakistani group (girls 0.63, $0.41-0.99$; boys $0.49,0.27-0.89$ ), as well as Black African boys $(0.10,0.02-0.38)$, Indian boys $(0.40,0.21-0.77)$, and Bangladeshi girls $(0.18,0.05-0.65)$, compared to their White peers. After adjusting for social support, participation, and adversity factors, significantly reduced odds for mental health problems remained only for Black African boys (OR 0.16, 0.04-0.72).

Conclusion Socioeconomic status (SES) confounds resilience factors against mental health problems apparent in young people from some ethnic minority groups. Despite greater socioeconomic disadvantage, there was reduced prevalence of mental health problems for these young people after adjustment for SES. Furthermore, the changes to ORs after adjusting for social support, participation, and adversity factors suggest ethnic inequalities in mental health outcomes in this sample could be partly explained by these social factors. Further analysis is needed to investigate mediating mechanisms operating here. Social interventions may help foster resilience in young people against mental health problems, irrespective of ethnicity.

\section{OP29 INTERACTION BETWEEN SOCIOECONOMIC POSITION AND SOCIAL INTEGRATION IN SUICIDE MORTALITY: A NATIONALLY REPRESENTATIVE COHORT STUDY}

${ }^{1} \mathrm{C} \mathrm{Kim}{ }^{*},{ }^{2}$ J Dunn. ${ }^{1}$ Health Policy, McMaster University, Hamilton, Canada; ${ }^{2}$ Department of Health Aging and Society, McMaster University, Hamilton, Canada

10.1136/jech-2019-SSMabstracts.29

Background A low level of social integration and lower socioeconomic position (SEP) are well-known risk factors for suicidal behavior. Most literature has suggested that the effects of family types as a proxy of social integration and SEP measures on suicide are merely additive. However, as social integration is not dependent on SEP, the association of these effects could be interactive. Since the protective effects of social integration vary by gender, so could this interaction. The aim of this study was to examine the interaction between social integration, SEP and gender on suicide mortality in the Canadian context.

Methods Using data from the 1991 Canadian Census Health and Environment cohort (CanCHEC) —which included 2.5 million Canadians over a 20-year follow up period - we applied Cox proportional hazards regression models to examine the association among living arrangements (lives alone versus single parent family versus others), education (secondary versus non-secondary), income (low income versus non-low income), and employment status (unemployed versus else) by gender. Models were developed to observe how living arrangements attenuated the association between SEP measures and suicide. In the full model, we added interaction terms between living arrangements and employment status.

Results In model 1, which was only adjusted for age and three SEP measures, both men and women with low income (Hazard Ratio (HR): 1.846 [women], HR: 1.278 [Men]) and who were unemployed (HR: 1.501 [Women], HR: 1.677 [Men]) were more likely to be exposed to completed suicide. In all models, lower education was associated with suicide risk among men, but not among women. In model 2, when living arrangements were added, the association between SEP measures and suicide was much attenuated among women, but not among men. In the full model, an interactive effect between unemployment and living arrangements (living alone) was not shown among men. However, there was a significant interactive effect for women, demonstrating that unemployed women who do not live alone were 1.429 times more likely to complete suicide than employed women living with others, but women living alone and unemployed were 2.125 times more likely to do so.

Conclusion While SEP had more independent impacts from social integration on suicide among men, there were significant synergetic effects on suicide mortality among women in Canada.

\section{OP30 WHAT HAPPENS AFTER SELF-HARM? AN EXPLORATION OF SELF-HARM AND SUICIDE USING THE NORTHERN IRELAND REGISTRY OF SELF-HARM}

${ }^{1}$ A Maguire, ${ }^{1} \mathrm{~F}$ Tseliou, ${ }^{2} \mathrm{D}$ O'Hagan, ${ }^{1} \mathrm{D}$ O'Reilly, ${ }^{1} \mathrm{~S}$ McKenna*. ${ }^{1}$ Centre for Public Health, Queen's University, Belfast, UK; ${ }^{2}$ Public Health Agency, UK

\subsection{6/jech-2019-SSMabstracts.30}

Rationale Suicide is a major public health concern and Northern Ireland (NI) has the highest rate of both self-harm and suicide in the UK and Ireland. In order to target prevention strategies effectively it is vital to understand who is most at risk. Those who present with self-harm offer a prime opportunity for intervention. The aim of this study is to examine the risk factors for completed suicide following presentation with self-harm.

Data The Northern Ireland Registry of Self-Harm (NIRSH) collects information on all self-harm and suicide ideation presentations to all Emergency Departments in NI. NIRSH data for the four years 2012-2015 was linked to centralised electronic data relating to primary care, social services and prescribed medication and mortality records.

Methods Initial analyses describing the profile of those who present with self-harm was followed by logistic regression to quantify the likelihood of mortality with adjustment for factors associated with mental ill health and suicide risk.

Results The cohort consisted of all 1,483,435 individuals born or resident in NI from 1st January 1970 until $31^{\text {st }}$ December 2015 (maximum age in 2015, 45 years). During the follow-up period, $11,371(0.8 \%)$ individuals presented with self-harm and $1,719(0.1 \%)$ died by suicide. Rates of self-harm were equivalent for males and females with highest rates observed in the 18-24 years age group, and more common in deprived than affluent areas $(\mathrm{OR}=3.34,95 \% \mathrm{CI} 3.12,3.57)$. Rates of self-harm was highest among those who were (or ever had been) in the care of social services (OR $=12.06,95 \% \mathrm{CI} 11.26$, 12.93). Most individuals self-harm via self-poisoning with psychotropic medications (70.9\%), followed by self-injury with a sharp object $(21.6 \%)$. Although only $142(1.3 \%)$ of those who presented with self-harm went on to die by suicide, in the unadjusted model those who self-harmed were almost 12 times more likely to die by suicide compared to those who did not present with self-harm $(\mathrm{OR}=11.79$, 95\%CI 9.92,14.01). Suicide was more likely in individuals who selfharmed with more violent methods such as strangulation or drowning. After full adjustment for gender, age, social services care, area of residence and deprivation individuals who selfharm are still 7 times more likely to die by suicide $(\mathrm{OR}=7.05$, 95\%CI 5.88,8.45). 
Further analysis is underway to explore which particular traits and characteristics of those who self-harm are most associated with risk of suicide using cox regression in order in inform intervention targeting.

\section{Thursday 5 September}

\section{Cancer 2}

\section{OP31 MEAT INTAKE AND CANCER RISK: PROSPECTIVE ANALYSES IN UK BIOBANK}

A Knuppel ${ }^{*}$, K Papier, PN Appleby, TJ Key, A Perez-Cornago. Cancer Epidemiology Unit, Nuffield Department of Population Health, University of Oxford, Oxford, UK

\subsection{6/jech-2019-SSMabstracts.31}

Background The latest meta-analysis from the World Cancer Research Fund/American Institute for Cancer Research concluded that red meat was a probable cause and processed meat a convincing cause of colorectal cancer. However, evidence for associations between red and processed meat intake and other cancer sites is limited. Furthermore, few studies have examined the association between poultry intake and cancer risk. Therefore, the aim of this study was to examine the associations between red, processed meat and poultry intake and incidence for 20 common cancer sites.

Methods We analysed data from 475,488 participants (54\% women) in UK Biobank. Participants were aged 37-73 years and cancer free at baseline. Cancer diagnosis and death due to cancer during follow-up were determined using data-linkage with cancer and death registries (with follow-up until 30 November 2014 for England and Wales and until 31 December 2014 for Scotland, respectively). Information on meat consumption was based on a touchscreen questionnaire completed at baseline covering type and frequency of meat intake. We used multivariable-adjusted Cox proportional hazards models to determine the association between baseline meat intake and cancer incidence. Analyses of lung cancer risk were restricted to never smokers. All analyses were adjusted for socio-demographic, lifestyle and women-specific factors.

Results Over a mean 5.7 (SD 1.1) years of follow-up 23,117 participants were diagnosed with any type of malignant cancer. Red meat intake was positively associated with colorectal cancer (Hazard ratio (HR) per $50 \mathrm{~g} /$ day increment in intake 1.20, 95\% Confidence Interval (CI) 1.02-1.41), breast cancer (1.13, 1.01-1.27) and prostate cancer $(1.14,1.00-1.29)$. Processed meat intake was positively associated with risk for colorectal cancer (HR per $20 \mathrm{~g}$ /day increment in intake 1.16, 95\% CI 1.04-1.30). Poultry intake was positively associated with risk for malignant melanoma (HR per $30 \mathrm{~g} /$ day increment in intake 1.20 , 95\% CI 1.00-1.44), prostate cancer $(1.11,1.02-$ 1.22) and non-Hodgkin lymphoma (1.26, 1.03-1.55).

Discussion Higher intakes of red and processed meat were associated with a higher risk of colorectal cancer. Red meat consumption was also positively associated with risk of breast and prostate cancer, but these associations are not supported by most previous prospective studies and may be affected by residual confounding. The positive associations of poultry intake with prostate cancer and non-Hodgkin lymphoma require further investigation.

\section{OP32 SOCIOECONOMIC STATUS AND BREAST CANCER MORTALITY IN SCOTLAND BY OESTROGEN RECEPTOR STATUS}

I Mesa-Eguiagaray*, S Wild, J Figueroa. Usher Institute of Population Health Sciences and Informatics, University of Edinburgh, Edinburgh, UK

\subsection{6/jech-2019-SSMabstracts.32}

Background Although breast cancer is a disease associated with high socioeconomic status (SES), previous studies report higher mortality amongst women with lower SES. Breast cancer prognosis is highly related to tumour characteristics, such as oestrogen receptor (ER) status. ER+ tumours have better prognosis than ER- tumours as they are responsive to hormone treatments. This study aims to examine if socioeconomic inequalities in breast cancer mortality in Scotland differ by ER status.

Methods All women diagnosed with breast cancer in Scotland (recorded in the cancer registry) from 1997 to 2016 were followed up to the end of 2016. Median follow up time was 5.5 years, and 5, 10 and 15 years Kaplan Meier estimates for all-cause mortality were calculated by Scottish Index of Multiple Deprivation (SIMD) -an area-based measure of deprivation, stratified by ER status $(82 \% \mathrm{ER}+)$. Cox models were used to estimate hazard ratios (HR) between women in the most deprived quintile (Q1) of areas and women in the least deprived quintile (Q5) of areas stratified by ER status. Models were adjusted for age, Scottish region, tumour characteristics (grade, tumour size and whether screen-detected or not), treatment regimens and Charlson index of comorbidity. The potential for SIMD and ER status interaction was tested using likelihood ratio test.

Results Among the total of 72,217 women with breast cancer $12,923(18 \%)$ were in Q1 and 14,980 (21\%) were in Q5. There were 5,688 (44\%) deaths in Q1 and 4,526 (30\%) deaths in Q5. Women in Q1 had more advanced tumours than women in Q5: $43 \%$ vs 37\% with poorly differentiated tumours, $45 \%$ vs $40 \%$ with tumours bigger than $2 \mathrm{~cm} 24 \%$ vs $30 \%$ screen detected tumours. Difference in proportions who were dead at 5, 10 and 15 years between Q1 and Q 5 were $11 \%, 14 \%$ and $17 \%$ respectively. Five and 10 years mortality rates were higher for ER- tumours than for ER+ but there were no differences in mortality at 15 years. Fully adjusted Cox regression models for mortality for Q1 compared to Q5 gave HR of 1.40 [95\% CI: 1.30-1.50]) for women with ER+ tumours and 1.35 [95\% CI: 1.19-1.53] for women with ERtumours There was no evidence of interaction between SIMD and ER status ( $\mathrm{p}$ value $=0.375$ ).

Conclusion Socio-economic status is inversely associated with breast cancer mortality in Scotland regardless of ER status. Future studies are needed to determine cause of death and the role of comorbidities in this population.

\section{OP33 BODY SIZE AND COMPOSITION IN RELATION TO RISK OF ENDOMETRIAL CANCER IN UK BIOBANK}

W Omiyale, NE Allen, S Sweetland*. Nuffield Department of Population Health, University of Oxford, Oxford, UK

\subsection{6/jech-2019-SSMabstracts.33}

Background Obesity, as reflected by a high body mass index (BMI), is a well-known risk factor for endometrial cancer. Whether more precise measures of body fat, such as body fat 